\title{
Slow dynamics simulation of power systems
}

\author{
KWOK W CHEUNG ${ }^{1}$ and JOE H CHOW ${ }^{2}$ \\ ${ }^{1}$ ESCA Corporation, Bellevue, WA 98004-1448, USA \\ ${ }^{2}$ Electrical, Computer, \& Systems Engineering Department, Rensselaer \\ Polytechnic Institute, Troy, NY 12180-3590, USA
}

\begin{abstract}
This paper investigates the simulation of slow dynamics in two-time-scale power systems. A new approach is proposed to obtain the slow dynamics by projecting the trajectory of the post-fault system onto its slow manifold. This is achieved by a nonlinear projection of the full order system initial condition onto the slow manifold, such that the fast intraarea dynamics are not excited. A projection scheme is developed and applied to two-test power systems.
\end{abstract}

Keywords. Slow dynamics simulation; power systems; post-fault system; slow manifold; singular perturbations.

\section{Introduction}

Transient stability studies provide information about the capability of a power system to remain in synchronism under disturbances such as sudden or sustained load changes, loss of generation and transmission facilities, or momentary faults (Kundur 1985). For a large power system with highly stressed power transfer on interties, the loss of synchronism usually is due to the slower interarea modes, that is, groups of machines pulling away from the rest of the system (Kundur et al 1990). If the noncritical fast inter-machine dynamics within the groups can be eliminated, then the analytical complexity can be significantly reduced.

This paper investigates the simulation of the slow dynamics in two-time-scale power systems modelled as nonlinear autonomous singularly perturbed systems

$$
\begin{aligned}
& \dot{x}=f(x, z, \mu), \quad x \in R^{n_{s}}, \\
& \mu \dot{z}=g(x, z, \mu), \quad z \in R^{n_{s}} \text {, }
\end{aligned}
$$

where " denotes the derivative with respect to the slow time-scale $t, \mu>0$ is the singular perturbation parameter, and the total system order is $n=n_{s}+n_{f}$. Given the initial condition $x_{0}=x\left(t_{0}\right), z_{0}=z\left(t_{0}\right)$, system (1), (2) generates a trajectory $x(t), z(t)$ from $t=t_{0}$ to $t=t_{f}$, which, in general, contains both slow and fast dynamics. Our objective is to obtain the slow components of $x(t), z(t)$.

A common approach is to explicitly construct reduced-order slow models (Peponides et al 1982; Chow 1982). However, for a large-scale power system, exact nonlinear 
slow subsystems cannot be readily obtained in an analytical form. Another approach is to use an implicit integration routine and large step sizes for simulation (deMello et al 1992). However, such simulations usually do not closely track the slow components of the system response.

In this paper, we propose to use a nonlinear slow-fast transformation (Cheung \& Chow 1991) to project a post-fault trajectory onto the slow manifold. If a point of the trajectory is projected onto the slow manifold by eliminating its fast component, then using this projected point as the initial condition for the full order system, the resulting simulation will not contain any fast dynamics. Furthermore, the trajectory obtained will be identical to the slow dynamics simulated from the exact reduced-order slow model. In this technique, no construction of the slow subsystem is required. Thus, existing transient simulation packages and models can be used to investigate interarea stability of power systems. Moreover, the elimination of the oscillations from the stable higher frequency modes speeds up the simulation process since larger stepsizes can be used in the simulation.

The remainder of the paper is organized as follows. Section 2 presents the integral manifolds and decomposition of singularly perturbed systems. In $\S 3$, the notion of the slow dynamics projection is introduced in terms of the slow and fast manifolds, and a slow dynamics projection scheme is developed. In $\$ 4$, the projection scheme is applied to two-test systems.

\section{Integral manifolds of singularly perturbed systems}

In this section we discuss the integral manifolds and the decomposition of nonlinear singularly perturbed systems (Sobolev 1984; Cheung \& Chow 1991) in the form of system (1), (2). It is assumed that system (1), (2) satisfies the following assumptions in a region $D \subset R^{n}$ :

(A1) All equilibria are isolated equilibrium points among which the origin, $x=0$, $z=0$, is the equilibrium point of interest.

(A2) Equation $g(x, z, 0)=0$ has an isolated solution $z=h^{(0)}(x)$ satisfying $h^{(0)}(0)=0$.

(A3) Functions $f, g$ and $h^{(0)}$ are $C^{2}$ for $\left|z-h^{(0)}(x)\right| \leqslant \rho_{0}, 0 \leqslant \mu \leqslant \mu_{0}$.

(A4) The eigenvalues $\lambda_{i}, i=1, \ldots, n_{f}$, of the matrix $(\partial g / \partial z)\left(x, h^{(0)}(x), 0\right)$ satisfy the inequality $\mathscr{R}_{e}\left\{\lambda_{i}\right\}<0$.

For a two-time-scale dynamical system, it is desirable to obtain two decoupled reduced-order models which govern the slow and the fast dynamics of the full-order system. The decoupling scheme starts by seeking specific hypersurfaces or integral manifolds in the $n$-dimensional region $D$ such that for any initial condition of the system on these manifolds, the solution of the system will remain on the manifolds thereafter. For singularly perturbed systems, invariant manifolds are formally defined as follows.

\section{DEFINITION 1}

An invariant manifold $\mathscr{M}_{\mu}$ for system (1), (2) is a manifold in $R^{n}$ such that if the initial condition $x_{0}=x\left(t_{0}\right), z_{0}=z\left(t_{0}\right)$ is on $\mathscr{M}_{\mu}$, the solution $(x(t), z(t))$ is on $\mathscr{M}_{\mu}$ for all $t>t_{0}$.

We will use two special types of invariant manifolds, namely, slow manifolds and fast manifolds, to decompose the slow and the fast dynamics of (1), (2), respectively. 
Under assumptions (A1)-(A4), it is well-known (Fenichel 1979) that a slow manifold exists and is defined by

$$
\mathscr{M}_{s}: z=h(x, \mu)
$$

where $h(x, \mu)$ satisfies the slow manifold equation

$$
g(x, h(x, \mu), \mu)=\mu(\partial h / \partial x) f(x, h(x, \mu), \mu),
$$

and is a $C^{2}$ function of $x$ and $\mu$. The dynamics on the slow manifold are governed by the $n_{s}$-dimensional system

$$
\dot{x}=f(x, h(x, \mu), \mu) \text {. }
$$

It follows from (A2) that $h(0, \mu)=0$, implying that the origin is an equilibrium point on $\mathscr{M}_{s}$.

If $z\left(t_{0}\right)=h\left(x\left(t_{0}\right), \mu\right)$, then the trajectory of $(1)$, (2) will remain on $\mathscr{M}_{s}$ for all $t>t_{0}$. Thus we measure the deviation $z$ from $\mathscr{M}_{s}$ by a new fast variable

$$
v=z-h(x, \mu) \text {. }
$$

Differentiating (6) with respect to the slow time-scale $t$ yields

$$
\begin{aligned}
\mu \dot{v} & =g(x, v+h(x, \mu), \mu)-\mu(\partial h / \partial x) f(x, v+h(x, \mu), \mu) \\
& \stackrel{\text { def }}{=} \tilde{g}(x, v, \mu) .
\end{aligned}
$$

Thus in the variables $x$ and $v$, system (1), (2) becomes

$$
\begin{aligned}
\dot{x} & =f(x, v+h(x, \mu), \mu), \\
\mu \dot{v} & =\tilde{g}(x, v, \mu) .
\end{aligned}
$$

Lemma 1. (Cheung \& Chow 1991) The function $\tilde{g}$ satisfies $\tilde{g}(x, 0, \mu)=0$, and $v=0$ is the slow manifold of the $(x, v)$-system $(8),(9)$.

Lemma 1 establishes that $v$ in (9) is the purely fast variable having no slow components as $\tilde{g}$ does not contain any $v$-independent terms. To eliminate the fast part of the slow variable $x$ in (8), we introduce the transformation

$$
x=u+\mu H(u, v, \mu),
$$

where $u$ is the purely slow variable, and $\mu H(u, v, \mu)$ is the fast part modulated by the slow variable $u$. Since $x$ is purely slow when $v=0, H(u, 0, \mu)=0$.

Differentiating $(10)$ with respect to $t$, we obtain

$$
\begin{aligned}
\dot{u}= & f(u+\mu H(u, v, \mu), v+h(u+\mu H(u, v, \mu), \mu), \mu) \\
& -\mu(\partial H / \partial u) \dot{u}-(\partial H / \partial v) \tilde{g}(u+\mu H(u, v, \mu), v, \mu) .
\end{aligned}
$$

If $u$ is the purely slow variable, then $u$ must satisfy the slow dynamical equation (5),

$$
\dot{u}=f(u, h(u, \mu), \mu) \stackrel{\operatorname{def}}{=} \hat{f}(u, \mu) .
$$


To ensure that $u$ is a slow variable, we equate (11) and (12) to obtain a partial differential equation for $H(u, v, \mu)$

$$
\begin{aligned}
f(u+\mu H(u, v, \mu), v+h(u+\mu H(u, v, \mu), \mu), \mu)-f(u, h(u, \mu), \mu) \\
=\mu(\partial H / \partial u) f(u, h(u, \mu), \mu)+(\partial H / \partial v) \tilde{g}(u+\mu H(u, v, \mu), v, \mu)
\end{aligned}
$$

with the boundary condition $H(u, 0, \mu)=0$.

Using the transformation (10), system (8), (9) decouples into

$$
\begin{aligned}
\dot{u} & =\hat{f}(u, \mu), \\
\mu \dot{v} & =\hat{g}(u, v, \mu),
\end{aligned}
$$

where $\hat{g}(u, v, \mu)=\tilde{g}(u+\mu H(u, v, \mu), v, \mu)$. In (14), $u$ is completely decoupled, and is all the slow part in $x$. In $(15)$, since $\hat{g}(u, 0, \mu)=0$, the meaning of the fast variable $v$ remains unchanged from that in (9), with $u$ acting as slowly time-varying coefficients in its dynamical equation.

Using (6) and (13), (10) can be rewritten to define the fast manifold

$$
\mathscr{H}_{f, \mu}: x-u=\mu H(u, z-h(x, \mu), \mu) .
$$

The existence and properties of $H$ to (13) is shown by Kelley (1967).

Note that (16) can be interpreted as a hypersurface in the $(x, z)$ state space parametrized by the variable $u$. The dependence of $\mathscr{H}_{f, u}$ on the slow variable $u$ implies that $\mathscr{M}_{\Gamma, u}$ is a slowly time-varying dynamic manifold in the $(x, 2)$ state space. In fact, any trajectory $(x(t), z(t))$ is a trajectory on $\mathscr{H}_{f . u(t)}$. In the special case where $u=u_{e}$ is an equilibrium of (14), $\mathscr{M}_{f, u_{\odot}}$ is a time-invariant fast manifold.

For linear singularly perturbed systems, the transformations (6) and (10) reduce to the block-diagonalization results (Kokotovic et al 1986). Consider system (1), (2) linearized about the equilibrium point $x=0, z=0$,

$$
\begin{gathered}
\dot{x}=A_{11} x+A_{12} z, \\
\mu \dot{z}=A_{21} x+A_{22} z,
\end{gathered}
$$

where $A_{22}$ is nonsingular. Transformation (6) becomes

$$
v=z-L(\mu) x, v \in R^{n_{f}}
$$

where $L(\mu)$ satisfies the matrix quadratic equation

$$
A_{21}+A_{22} L-\mu L\left(A_{11}+A_{12} L\right)=0
$$

which is obtained from the slow manifold equation (4). The $(x, v)$-system (8), (9) reduces to

$$
\begin{aligned}
& \dot{x}=\left(A_{11}+A_{12} L\right) x+A_{12} v, \\
& \mu \dot{v}=\left(A_{22}-\mu L A_{12}\right) v .
\end{aligned}
$$

The slow variable transformation $(10)$ is

$$
x=u+\mu N(\mu) v, \quad u \in R^{n},
$$


where $N(\mu)$ satisfies the matrix equation

$$
\mu\left(A_{11}+A_{12} L\right) N-N\left(A_{22}-\mu L A_{12}\right)+A_{12}=0,
$$

derived from the fast manifold equation (13). The $(u, v)$-system (14), (15) reduces to

$$
\begin{gathered}
\dot{u}=\left(A_{11}+A_{12} L\right) u, \\
\mu \dot{v}=\left(A_{22}-\mu L A_{12}\right) v .
\end{gathered}
$$

In contrast to nonlinear systems, the fast subsystem (26) is independent of the purely slow variable. It is also of interest to note that $\mathscr{H}_{s}$ and $\mathscr{H}_{f, 0}$ are generalizations of the slow and the fast eigensubspaces in linear systems.

The decompositicn of the slow and fast dynamics of nonlinear singularly perturbed systems rely on the solutions of $h$ and $H$ of the slow manifold equation (4) and the fast manifold equation (13), respectively, which are both formulated in terms of partial differential equations. Using asymptotic methods, approximate solutions for both the slow and the fast manifold equations can be obtained (Cheung 1991). For linear singularly perturbed systems, the solutions of the slow and the fast manifolds reduce to solving two sets of algebraic equations (20) and (24). Since $A_{22}$ is invertible, the contraction mapping technique (Kokotovic 1975) can be used to establish a $\mu^{*}>0$ such that the solutions $L$ and $N$ of (20) and (24), respectively, exist for $0<\mu \leqslant \mu^{*}$. Moreover, $L$ and $N$ can be computed from asymptotic expansions through the recursions

and

$$
L_{k+1}=-A_{22}^{-1}\left[A_{21}-\mu L_{k}\left(A_{11}+A_{12} L_{k}\right)\right]
$$

$$
N_{k+1}=A_{12}+\mu\left[N_{k} L_{k} A_{12}+\left(A_{11}+A_{12} L_{k}\right) N_{k}\right] A_{22}^{-1}, \quad k=1,2, \ldots
$$

At each iteration, $L$ and $N$ are approximated by $L_{k}$ and $N_{k}$, respectively, to obtain the approximate slow eigensubspace $z=L_{k} x$ and fast eigensubspace $x=\mu\left(I_{n_{g}}-\mu N_{k} L_{k}\right)^{-1}$ $N_{k} z$. The recursions (27) and (28) will be used in the slow dynamics projection scheme proposed in the next section.

\section{Slow dynamics projection}

The results in the previous section illuminate two approaches to the simulation of the slow dynamics in the system (1), (2). In the first approach, the slow subsystem (14) can be constructed. However, the transformations (6) and (10) can seldom be obtained in exact analytical form. As a result, the slow subsystem (14) is usually approximated asymptotically using the first few terms of the series expansions of $h$ and $H$.

In the second approach, the transformations (6) and (10) can be applied to the $(x, z)$ system $(1),(2)$, to project the variables $(x, z)$ onto the slow manifold $\mathscr{M}_{s}$. To project a trajectory $(x(t), z(t))$ onto $\mathscr{H}_{s}$, it is not necessary to carry out the projection operation for every point of the trajectory. Once a trajectory lies on $\mathscr{H}_{s}$, it will stay on $\mathscr{H}_{s}$ thereafter. Thus, the low dynamics projection of a trajectory onto $\mathscr{H}_{\mathrm{y}}$ can be formed by projecting one single point, for example, its initial condition, onto $\mathscr{M}_{s}$ and the slow dynamics can be obtained by simulating the $(x, z)$ system (1), (2), using the 
projected initial condition. Knowing $h$ and $H$, the initial condition $\left(u_{0}=u\left(t_{0}\right)\right.$, $\left.v_{0}=v\left(t_{0}\right)\right)$ of the $(u, v)$ system (14), (15) can be computed from the initial condition $\left(x_{0}=x\left(t_{0}\right), z_{0}=z\left(t_{0}\right)\right)$ of the $(x, z)$ system (1), (2). Then $v_{0}$ is set to zero to obtain the projected initial condition

$$
x_{p}=u_{0}, \quad z_{p}=h\left(x_{p}, \mu\right),
$$

of the $(x, z)$ system.

Although the projection of the initial condition still involves the expressions for $h$ and $H$, it can now be computed numerically. To avoid solving for the partial differential equations, we propose a projection scheme based on the slow subspace of the linearized model of (1), (2). We first summarize the slow subspace projection.

For linear singularly perturbed systems, the slow dynamics projection reduces to the projection along the fast eigensubspace onto the slow eigensubspace. Consider again the linearized system (18). The two-stage transformation for nonlinear systems reduces to a block-diagonalized transformation

$$
T=\left[\begin{array}{lc}
I_{n_{s}} & \mu N \\
L & I_{n_{f}}+\mu L N
\end{array}\right]
$$

where $L$ and $N$ satisfy the matrix equations (20) and (24), respectively. The slow dynamics projection then reduces to a series of linear operations such that for any given point $(x, z)$, its projection becomes

$$
\begin{aligned}
{\left[\begin{array}{l}
x_{p} \\
z_{p}
\end{array}\right] } & =T\left[\begin{array}{ll}
I_{n_{s}} & 0 \\
0 & 0
\end{array}\right] T^{-1}\left[\begin{array}{l}
x \\
z
\end{array}\right] \\
& =\left[\begin{array}{ll}
I_{n_{s}}+\mu N L & -\mu N \\
L\left(I_{n_{s}}+\mu N L\right) & -\mu L N
\end{array}\right]\left[\begin{array}{l}
x \\
z
\end{array}\right] \stackrel{\operatorname{dsl}}{=} \mathscr{P}_{L}\left[\begin{array}{l}
x \\
z
\end{array}\right] .
\end{aligned}
$$

The projection $\mathscr{P}_{L}$ can be computed asymptotically using the recursions (27) and (28). It is a key step in the following algorithm to obtain the slow dynamics of a trajectory $x(t), z(t)$ from $t=t_{0}$ to $t=t_{f}$.

Algorithm SDP: Slow dynamics projection algorithm

Step 1: Linearize the system (1), (2) about the origin and obtain the state matrices $A_{11}, A_{12}, A_{21}$ and $A_{22}$ of (18).

Step 2: Set $k=0$ and let $L_{\mathbf{k}}$ and $N_{k}$ be null matrices of appropriate dimensions.

Step 3: Use the recursions (27) and (28) to update $L_{k+1}$ and $N_{k+1}$.

Step 4: Repeat step 3 until $L_{k+1}$ and $N_{k+1}$ converge.

Step 5: Integrate system (1), (2) forward in time using the initial condition $\left(x_{0}, z_{0}\right)$ to a sufficiently long period of time $t_{p}<t_{f}$ to obtain $\left(x\left(t_{p}\right), z\left(t_{p}\right)\right)$.

Step 6: Project $\left(x\left(t_{p}\right), z\left(t_{p}\right)\right)$ using (31) to obtain $x_{p}\left(t_{p}\right), z_{p}\left(t_{p}\right)$.

Step 7: If $t_{p}=t_{0}$, go to step 10; otherwise, go to step 8 .

Step 8: Integrate system (1), (2) backward for one integration time step $t_{s}$.

Step 9: Let $t_{p} \leftarrow t_{p}-t_{s}$ and go to step 6 .

Step 10: Integrate system (1), (2) forward in time from $t=t_{0}$ to $t=t_{f}$ using $x_{p}\left(t_{0}\right)$, $z_{p}\left(t_{0}\right)$ as the initial condition. 
Algorithm SDP is based on two assumptions:

(I) The slow eigensubspace is a good approximation of the slow manifold $\mathscr{H}_{s}$.

(II) The fast eigensubspace is a good approximation of the fast manifold $\mathscr{M}_{f, u_{0}}$ corresponding to the states at $t=t_{p}\left(x\left(t_{p}\right)=u\left(t_{p}\right)+\mu H\left(u\left(t_{p}\right), v\left(t_{p}\right), \mu\right), z\left(t_{p}\right)=\right.$ $\left.v\left(t_{p}\right)+h\left(x\left(t_{p}\right), \mu\right)\right)$.

If the initial condition $\left(x_{0}, z_{0}\right)$ is close to the slow manifold $\mathscr{M}_{S}$, then one can set $t_{p}=t_{0}$ and skip step 5 and steps 7 through 9. For initial conditions far from $\mathscr{M}_{S}$, assumption (II) is not valid. The projected initial condition contains some significant errors due to the approximation of the fast manifold, even though the projected initial condition is very close to the slow manifold. Since the slow manifold is attractive from assumption (A4), step 5 will bring the system state closer to the slow manifold so that projection errors due to fast manifold approximation are reduced. Step 6 will force the system trajectory to 'slide' on the slow eigensubspace in the backward-in-time integration and present a fast instability in backward time.

\section{Interarea modes of two-time-scale power systems}

In large power systems, groups of strongly connected machines often exhibit coherent slow motions. This physical phenomenon is attributed to the connections within the coherent groups being stiffer or more dense than the connection between the coherent groups (Chow 1982; Chow \& Kokotovic 1985). Consider the following $n$-machine power system modelled by Anderson \& Fouad (1977)

$$
\begin{array}{r}
m_{1} \ddot{\delta}_{i}=P_{i}-D_{i} \dot{\delta}_{i}-\sum_{j=1, j \neq i}^{n}\left[C_{i j} \sin \left(\delta_{i}-\delta_{j}\right)+E_{i j} \cos \left(\delta_{i}-\delta_{j}\right)\right], \\
i=1, \ldots, n,
\end{array}
$$

where the subscript $i$ denotes the variables associated with the $i$ th machine, and

$$
\begin{aligned}
m_{i} & =\text { inertia constant, } \delta_{i}=\text { rotor angle, } \\
P_{i} & =P_{m i}-\left|V_{i}\right|^{2} G_{i i}, \quad P_{m i}=\text { mechanical input power, } \\
C_{i j} & =V_{i} V_{j} B_{i j}, \quad E_{i j}=V_{i} V_{j} G_{i j}, D_{i}=\text { damping coefficient, } \\
V_{i} & =\text { constant voltage behind machine transient reactance, } \\
B_{i j} & =\text { transfer susceptance in the reduced } Y \text { matrix, } \\
G_{i j} & =\text { transfer conductance in the reduced } Y \text { matrix. }
\end{aligned}
$$

We assume the power system model (32) to consist of $r$ slow coherent areas, and denote by a small parameter $\varepsilon>0$ the ratio of the strength of the external connections between machines in different areas to the strength of internal connections between machines in the same areas. Let $J_{\alpha}$ be the index set of all machines $i$ in area $\alpha$, and $\Sigma_{i}^{\alpha}$ denote the summation over all machines $i \in J_{\alpha}$. To exhibit the $\varepsilon$-weak connections, (32) is rewritten as

$$
\begin{aligned}
& m_{i} \ddot{\delta}=P_{i}(\varepsilon)- \sum_{j}^{\alpha}\left[C_{i j}^{I} \sin \left(\delta_{i}-\delta_{j}\right)+E_{i j}^{I} \cos \left(\delta_{i}-\delta_{j}\right)\right] \\
&-\varepsilon\left\{D_{i}^{\alpha} \dot{\delta}_{i}+\sum_{\beta \neq \alpha}^{r} \sum_{j}^{\beta}\left[C_{i j}^{E} \sin \left(\delta_{i}-\delta_{j}\right)+E_{i j}^{E} \cos \left(\delta_{i}-\delta_{j}\right)\right]\right\}, \\
& i=1, \ldots, n,
\end{aligned}
$$


where the superscripts $I$ and $E$ denote "internal" and "external", respectively. The scaling of $D_{i}^{\alpha}$ by $\varepsilon$ is to delineate the fact that damping is relatively small.

To exhibit the time-scale property of the power system, we can cast (33) in a standard singularly perturbed form (Kokotovic et al 1986) using the slow coherency transformation (Chow 1982). The essence of this transformation is to introduce the inertia-weighted average of the machine angles in the same areas as the slow aggregate angle variable $\delta_{a}$ and the difference of the machine angles with respect to the reference machine in the area as the fast difference angle variable $\delta_{d}$. A detailed derivation of the standard singularly perturbed form model can be found in Chow (1982) and Cheung (1992).

Algorithm SDP is coded using functions from the Power System Toolbox (Chow \& Cheung 1992), a Matlab-based power system analysis and design package. It is applied to the simulation of the slow dynamics of a 3-machine, 10-bus system, and a 16-machine, 68-bus system. The simulation consists of the following procedure. The loadflow solution of the pre-fault system is first obtained to initialize the state variables of the machine models. All loads are modelled as constant impedance. Second and third order Runge-Kutta formulas with variable stepsizes are used to integrate the power system dynamic equations. The grouping algorithm (Chow 1982; Chow \& Cheung 1992) is used to identify the slow coherent areas in the system. Then the slow coherency transformation is used to cast the power system model in its singularly perturbed form. The slow dynamics projection algorithm SDP is used to provide the projection of the initial condition of the post-fault system.

\subsection{3-machine 10-bus system}

In the 3-machine 10-bus power system (US DOE Report 1987), machines 2 and 3 form a slow coherent group and machine 1 is a single-machine coherent group. Algorithm SDP with $t_{p}=t_{0}$ is used to simulate the slow dynamics following a $0.3 \mathrm{~s}$ duration three-phase to ground fault at bus 7. The relative angle plots of machines 2 and 3 with respect to machine 1 for the full order initial condition simulation and the projected initial condition simulation are given in figures $1 \mathrm{a}$ and $\mathrm{lb}$, respectively, showing that the slow dynamics projection successfully removes almost all the fast oscillations between machines 2 and 3 and retains the interarea oscillations. Note the discontinuities in the angles in figure $1 \mathrm{~b}$ when the fault is cleared and the initial condition projection is carried out. In this case, assumption (II) is satisfied, and algorithm SDP is essentially a linear projection.

\subsection{6-machine 68-bus system}

The 16-machine system (Schulz et al 1974) is a simplified model of the US Northeastern system. The New England system is represented in detail with machines 1. to 9, while the neighbouring utilities in New York, Pennsylvania and Michigan are modelled with large equivalent machines 10 to 16 . Based on the grouping algorithm, machines 1-9 form a slow coherent area (area 1) while machines 10-16 form the other coherent area (area 2).

Algorithm SDP is used to simulate the slow dynamics following a $0.21 \mathrm{~s}$ duration three-phase to ground-fault at bus 2, which is cleared by tripping the line between bus 1 and bus 2 . The full order initial condition simulation of the angles of machines 1 to 9 is given in figure $2 \mathrm{a}$. The simulation using the projected initial condition from 

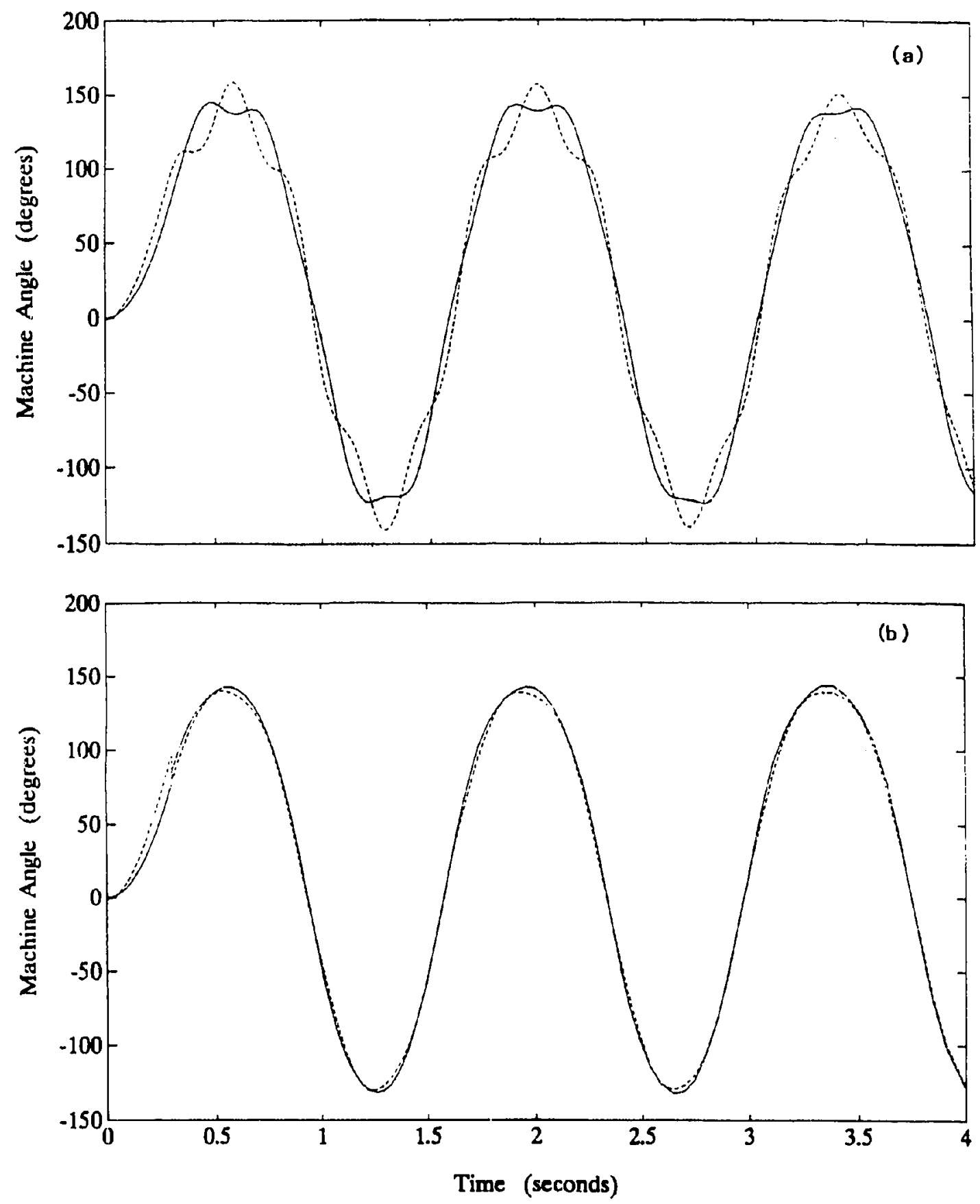

Figure 1. Interarea mode of 3-machine 10-bus system. Initial conditions - full order (a); from algorithm SDP with $t_{p}=t_{0}(\mathbf{b})$.

algorithm SDP with $t_{p}=t_{0}$ is shown in figure $2 \mathrm{~b}$. Note that the simulation does not contain any observable fast dynamics, implying that the projected initial condition is very close to the slow manifold. However, the slow dynamics in figure $2 \mathrm{~b}$ is about 0.5 second out of phase with the trajectories shown in figure $2 \mathrm{a}$, implying that the initial condition has been projected to an incorrect point on the slow manifold. Thus the results show that assumption (II) is not valid, and a linear projection does not provide sufficient accuracy.

Then algorithm SDP is applied with $t_{p}=t_{0}+2$ seconds. The full system state is projected onto the slow manifold and the system integrated backward in time to 

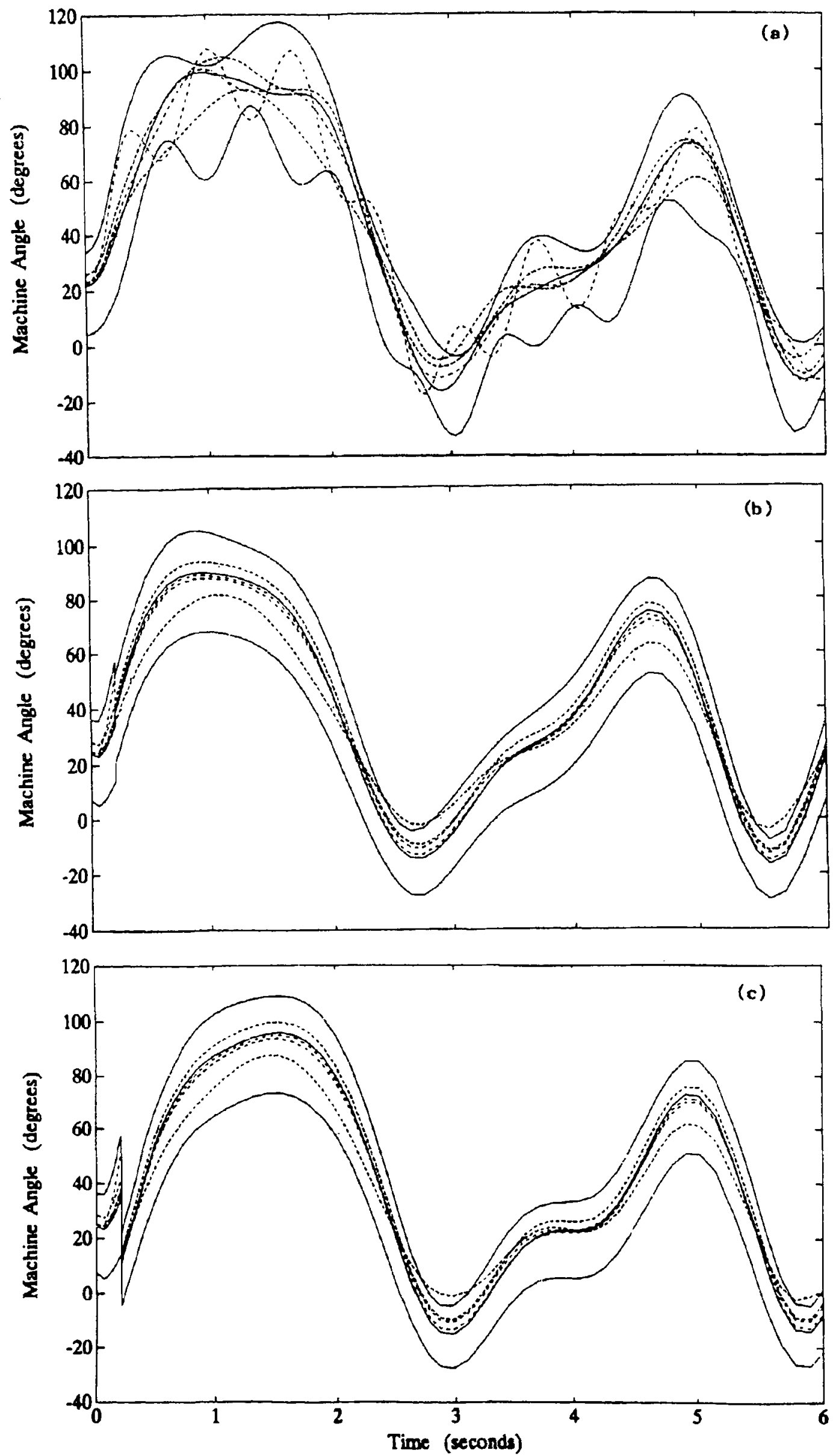

Figure 2. Interarea mode of 16-machine 68-bus system. Initial conditions - full order (a); from algorithm SDP with $t_{p}=t_{0}$ (b) and $t_{p}=t_{0}+2$ (c). 
find the projected initial condition. The simulation is shown in figure $2 \mathrm{c}$. Note the more substantial jump in the angles due to the projection when the fault is cleared. Also the phase of the slow dynamics now matches those of figure $2 \mathrm{a}$. The results show that the whole New England system, acting as a group, is swinging against the rest of the system. This is consistent with the slow coherency grouping technique (Chow 1982). Figure 2c shows very clearly the interarea oscillations of area 1 with respect to area 2 while the higher frequency oscillations within area 1 are suppressed significantly. The results here clearly indicate the existence of a nonlinear slow manifold in the swing equations of power systems. The simulation using the projected initial condition from algorithm SDP is almost $130 \%$ faster than that of the full order initial condition simulation because of the larger stepsizes being used by the variable stepsize integration routine. However, the computation of the initial condition $\left(x_{p}\left(t_{0}\right), z_{p}\left(t_{0}\right)\right)$ is time-consuming because of the projection required in every step of the backwardin-time integration. In practice, the backward-in-time integration can be avoided if one is interested in only eliminating the fast dynamics for $t>t_{p}$. In this case, the simulation can continue using the projected slow variables at $t=t_{p}$ (deMello et al 1982).

\section{Conclusions}

In this paper we have studied the slow manifold simulation of two-time-scale power systems using a projection method. The method is based on a nonlinear decoupling transformation which defines the slow and fast manifolds. A projection scheme is proposed for extracting the purely slow dynamics of a singularly perturbed system. The algorithm is applied to the simulation of the interarea modes of two power systems. The simulation results show that the irrelevant higher frequency dynamics are almost completely eliminated while the interarea modes are retained. Furthermore, the idea of projection may also be applied to define slow and fast energies which could be useful in direct stability analysis of power systems.

This research is supported in part by US NSF grants EC-8915667 and EC-9215076.

\section{References}

Andersen P M, Fouad A A 1977 Power system control and stability (Ames, IA: Iowa State University Press)

Cheung K W 1991 Manifold and stability analysis of singularly perturbed systems with applications to power networks, Ph D thesis, Rensselaer Polytechnic Institute, New York

Cheung K W, Chow J H 1991 Stability analysis of singularly perturbed systems using slow and fast manifolds. Proceedings of the 1991 American Control Conference, pp. 1685-1690

Chow J H (ed.) 1982 Time-scale modeling of dynamic networks with applications to power systems (New York: Springer-Verlag)

Chow J H, Cheung K W 1992 A toolbox for power system dynamics and control engineering education and research IEEE Trans. Power Syst. 7: 1559-1564

Chow J H, Kokotovic P V 1985 Time-scale modeling of sparse dynamic networks. IEEE Trans. Autom. Control AC-30: 714-722 
deMello F P, Feltes J W, Laskowski T F, Oppel L J 1992 Simulating fast and slow dynamic effects in power systems. IEEE Comput. Appl. Power 5: 33-38

Fenichel $\mathrm{N} 1979$ Geometric singular perturbation theory of ordinary differential equations. $J$. Differential Equations 31: 53-98

Kelley A 1967 The stable, center-stable, center, center-unstable, unstable manifolds. $J$. Differential Equations 3: 546-570

Kokotovic P V 1975 A Riccati equation of block-diagonalization of ill-conditioned systems. IEEE Trans. Autom. Control AC-20: 812-814

Kokotovic P V, Khalil H K, O'Reilly J 1986 Singular perturbation methods in control: Analysis and design (London: Academic Press)

Kundur P 1985 Evaluation of methods for studying power system stability. Proceedings of the International Symposium on Power System Stability, Ames, Iowa

Kundur P, Rogers G J, Wong D Y, Wang L, Lauby M G 1990 A comprehensive computer program package for small signal stability analysis of power systems. IEEE Trans. Power Syst. 5: 1076-1083

Peponides G, Kokotovic P V, Chow J H 1982 Singular perturbations and time scales in nonlinear models of power systems: IEEE Trans. Circuits Syst. CAS-29: 758-767

Schulz R P, Turner A E, Ewart D N 1974 Long term power system dynamics. EPRI Report 90-7-0, Palo Alto, California

Sobolev V A 1984 Integral manifolds and decomposition of singularly perturbed systems. Syst. Control Lett. 5: 169-179

US DOE Report 1987 Development of a decoupling methodology for on-line detection of system instabilities. Systems Development and Engineering Department, General Electric Company, Schenectady, New York 